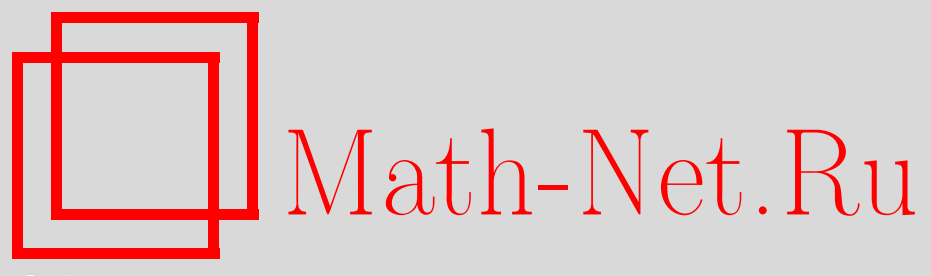

А. Н. Магазинов, Гипотеза Вороного для удлинений параллелоэдров Вороного, УМН, 2014, том 69, выпуск 4, 185-186

DOI: https://doi.org/10.4213/rm9601

Использование Общероссийского математического портала Math-Net.Ru подразумевает, что вы прочитали и согласны с пользовательским соглашением http://www . mathnet.ru/rus/agreement

Параметры загрузки:

IP : 44.207 .124 .84

26 апреля 2023 г., 11:42:31

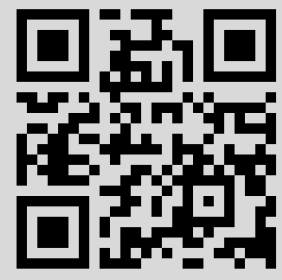




\section{Гипотеза Вороного для удлинений параллелоэдров Вороного}

\section{А. Н. Магазинов}

1. Определения и обозначения. Параллелоэдром (см. [1]) называется выпуклый многогранник $P$, допускающий разбиение $T(P)$ грань-в-грань аффинного пространства своими транслятами (параллельными копиями).

Пусть $\Lambda$ - $d$-мерная решетка в $\mathbb{R}^{d}, \mathbf{0} \in \Lambda$, а $\Omega$ - положительно определенная квадратичная форма от $d$ переменных. Тогда многогранник

$$
P_{V}=P_{V}(\Lambda, \Omega)=\left\{\mathbf{y} \in \mathbb{R}^{d}: \mathbf{y}^{T} \Omega \mathbf{y}=\min _{\mathbf{x}^{\prime} \in \Lambda}\left(\mathbf{y}-\mathbf{x}^{\prime}\right)^{T} \Omega\left(\mathbf{y}-\mathbf{x}^{\prime}\right)\right\}
$$

называется параллелоэдром Вороного решетки $\Lambda$ относительно формы $\Omega$.

Г. Ф. Вороной [2] сформулировал гипотезу о том, что каждый параллелоэдр является параллелоэдром Вороного. Полное доказательство или опровержение гипотезы Вороного остается открытой проблемой. Тем не менее для многих важных специальных классов параллелоэдров гипотеза Вороного верна (см. [2]-[5]).

Рассмотрим специальный класс параллелоэдров - параллелоэдры вида $P+I$, где $P$ - параллелоэдр, $I$ - отрезок, а под сложением понимается взятие суммы Минковского. Параллелоэдр вида $P+I$ назовем удлинением параллелоэдра $P$. Заметим, что не для всякой пары параллелоэдра $P$ и отрезка $I$ сумма Минковского $P+I$ является параллелоэдром.

В работе [6] В. П. Гришухин доказал гипотезу Вороного для удлинений $(d-2)$-примитивных параллелоэдров. Эти параллелоэдры, в силу результата Житомирского [3], образуют подкласс класса параллелоэдров Вороного. В [6] поставлена также задача доказать гипотезу Вороного для всех параллелоэдров вида $P+I$, где $P$ - параллелоэдр Вороного.

В данной работе дается набросок доказательства гипотезы Вороного для удлинений параллелоэдров Вороного. Таким образом, решается задача В. П. Гришухина и усиливается результат Р. Эрдала [4] о том, что гипотеза Вороного верна для зонотопов, являющихся параллелоэдрами.

Дадим необходимые определения.

Если $P$ - параллелоэдр, а $I$ - такой отрезок, что $P+I$ - также параллелоэдр, то направление отрезка $I$ назовем свободным для параллелоэдра $P$ (см., например, $[6])$.

Следуя [5], скажем, что параллелоэдр $P$ приводим, если он представим в виде прямой суммы параллелоэдров меньшей размерности: $P=P_{1} \oplus P_{2}$ (т. е. такой суммы Минковского, что линейные оболочки слагаемых $P_{1}$ и $P_{2}$ являются дополнительными подпространствами).

Для имеющего центр симметрии многогранника $Q$ обозначим его центр через $c(Q)$. Фасетным вектором гиперграни $F$ параллелоэдра $P$ называется вектор $2 c(F)-2 c(P)$ (центральная симметрия многогранников $P$ и $F$ следует из работы Г. Минковского [7]).

Будем говорить, что $d$-мерный параллелоэдр $P$ имеет крест, покрывающий фасетные векторы, если в $\mathbb{R}^{d}$ существуют две гиперплоскости $\alpha_{1}, \alpha_{2}$ такие, что каждый фасетный вектор параллелоэдра $P$ параллелен хотя бы одной из этих гиперплоскостей.

2. Основные результаты. Основным результатом работы является следующая теорема.

Теорема 1 [8]. Пусть $P$ и $P+I$ - параллелоэдры, причем $P$ - параллелоэдр Вороного. Тогда и $P+I-$ параллелоэдр Вороного.

Следующие утверждения являются ключевыми при доказательстве теоремы 1.

DOI: $10.4213 / \mathrm{rm} 9601$ 
Теорема 2. Пусть параллелоэдр Вороного Р имеет бесконечное множество свободных направлений. Тогда Р приводим.

Теорема 3. Пусть $P$ - параллелоэдр Вороного и имеет крест, покрывающий фасетные векторы. Тогда Р приводим.

Приведем схему доказательства теоремы 1.

Сначала устанавливается эквивалентность теорем 1 и 2. Отметим, что утверждение о том, что теорема 1 следует из теоремы 2 , приведено (хотя и без доказательства) в [9; теорема 3.18].

Далее доказываем теорему 2 индукцией по размерности параллелоэдра $P$. Одновременно с теоремой 2 доказывается и теорема 3.

Обозначим утверждение “теорема 2 верна для всех параллелоэдров Р размерности не более $d$ " через $\mathrm{A}(d)$. Аналогично определим утверждения $\mathrm{B}(d)$ для теоремы 3.

Основание индукции: утверждение $\mathrm{A}(d)$ верно при $d \leqslant 4$, утверждение $\mathrm{B}(d)$ верно при $d \leqslant 2$. Это мгновенно следует из того, что известна классификация всех параллелоэдров до размерности 4 включительно (см. [10]).

Собственно индукция осуществляется по следующей схеме:

$$
\cdots \Longrightarrow \mathrm{B}(n) \Longrightarrow \mathrm{A}(n+2) \Longrightarrow \mathrm{B}(n+1) \Longrightarrow \mathrm{A}(n+3) \Longrightarrow \cdots .
$$

Таким образом, для любого натурального $d$ будут доказаны утверждения $\mathrm{A}(d)$ и $\mathrm{B}(d)$. Следовательно, будут доказаны теоремы 2 и 3.

Поскольку верна теорема 2 , верна и теорема 1.

\section{Список литературы}

[1] Е. С. Фёдоров, Начала учения о фигурах, Тип. Имп. АН, СПб., 1885, 279 с.; Изд-во АН CCCP, M.-Л., 1953, 409 c. [2] G. F. Voronoï, J. Reine Angew. Math., 134 (1908), 198-287; 136 (1909), 67-181. [3] O. Zitomirskij, Журн. Ленингр. физ.-матем. об-ва, 2:2 (1929), 131-151. [4] R. M. Erdahl, European J. Combin., 20:6 (1999), 527-549. [5] A. Ordine, Proof of the Voronoi conjecture on parallelotopes in a new special case, Thesis (Ph.D.), Queen's University, Canada, 2005, 131 с. [6] В. П. Гришухин, Матем. сб., 197:10 (2006), 15-32; англ. пер.: V. P. Grishukhin, Sb. Math., 197:10 (2006), 1417-1433. [7] H. Minkowski, Gött. Nachr., 1897, 198-219. [8] A. Magazinov, Voronoi's conjecture for extensions of Voronoi parallelohedra, 2013, 27 pp., arXiv: 1308.6225. [9] A. Végh, Rácsok, kör-és gömbelrendezések, Thesis (Ph.D.), BME, Budapest, 2006, 115 c., http://www.doktori.hu/index.php?menuid=193\&vid=9821\&nyita=N\&lang=EN. [10] B. N. Delaunay, Изв. АН СCCP. VII сер. Отд. физ.-матем. наук, 1929, №1, 79-110; № 2, 147-164.

\section{Александр Николаевич Магазинов} (Aleksandr N. Magazinov)

Математический институт им. В. А. Стеклова РАН

E-mail: magazinov-al@yandex.ru
Представлено В. М. Бухштабером Принято редколлегией 28.03.2014 